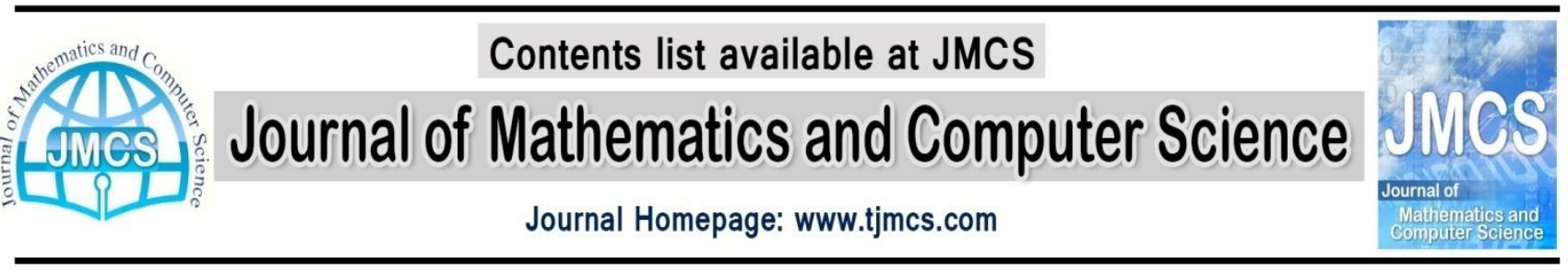

\title{
Using Hybrid Metaheuristics Algorithm to Balancing Bicycle Sharing System
}

\author{
Farzaneh Fadaie$^{1}$, Seyyed Yaser Bozorgi Rad $^{2}$ \\ ${ }^{1}$ Department of Computer Engineering, Babol Branch, Islamic Azad University, Babol, Iran. \\ Fadaie818@gmail.com \\ ${ }^{2}$ Assistant Professor Islamic Azad University of Babol.
}

Article history:

Received July 2015

Accepted August 2015

Available online August 2015

\begin{abstract}
Bike sharing system Balancing is an difficult and important issue due to the increasing popularity of this type of transportation, so we are dealing with a routing bicycles problem. In this system, there are several stations that bicycles are rented to individuals. The number of bicycles that can be embedded at each station with the same station's capacity is limited. The aim is to find the shortest possible time to start traveling from one station to the final destination station. In this article we study bike sharing system balancing problem in the real world (BBSP) by hybrid meta-discovery approach. Simulation results indicate an increase in the efficiency and speed of convergence rather than the earlier work.
\end{abstract}

Keywords: Bike sharing systems, Limitation programming, Particles mass, Optimization.

\section{Introduction}

Bike sharing system based on the balance in the bicycle distribution from one station to other. We want to maximize the system balance and every bicycle travels on the shortest route. System Balancing is related to find a route for each bicycle, including loading and unloading instruction in each station. The cost function must be designed to minimize the sum of the deviations in each station to the destination that these values including traveled distance and the activity of each bike in the whole system [Fadaie, 2015]. Limitation Programming is a powerful paradigm for solving hybrid search techniques, is used in artificial intelligence, operational research, algorithms, graph theory, and elsewhere in a broad spectrum. In computer science, limitation programming is programming paradigms in which expresses the relationship between the variables in the form of limitation. Programming limitation can be expressed in the form of a logical programming of limitation that are applied limitation to a logical plan. Flock optimized a specific objective function (here limitation satisfaction). Every agent considers (pbest) as best value and its position as s. This analogy information is each agent' personal experience. In addition, each agent considers (gbest) as the best 
value obtained so far in the group. The data is the deduction of the knowledge of how other factors of its surrounding apply. Each agent tries to change its position using the following information:

Current location s, the current speed v, pbest, gbest.

This modification can be expressed with the speed concept. The speed of each agent can be obtained from the following equation:

$$
v_{i}^{k+1}=w v_{i}^{k}+c_{1} \text { rand }_{1} \times\left(\text { pbest }_{i}-s_{i}^{k}\right)+c_{2} \operatorname{rand}_{2} \times\left(\text { gbest }-s_{i}^{k}\right)
$$

Where $v_{i}^{k}$ is speed of agent in $\mathrm{k}^{\text {th }}$ frequency, $w$ is Weight Function and $c_{j}$ is a weight coefficient. Rand is a random number between 0 and $1 ; s_{i}^{k}$, current agent location $i$ in $\mathrm{k}$ th frequency, is pbesti ، pbest of point $i$ and gbest is group gbest.

Definition of the right side (1) can be expressed as follows. Right (1) has three sentences. The first sentence is the agent' previous speed. The second and third sentences is to change agent' speed. Without the second and third sentences, flight agent will be continued in its previous direction to encounter with border. Agent tries to seek new areas and therefore, first sentences is search process corresponding diversity.

In other word, without first sentence, agent' flight speed is determined using only the current position and the best position in the past. Agent will try to converge to pbest or gbest.

\section{Bike Sharing System}

Bike sharing theory is designed to provide bicycle to citizens in the station that are embedded in the world. At each station, the bicycles are situated in positions so that users can easily take bike and return back to the station. However, stations will be often filled and be empty quickly as a result users are upset because they cannot take away bike or return bike. To avoid this problem, stations must be balanced. bike sharing system are balanced with the bicycle distribution, using specific devices .So , balancing system related to the vehicle to find a tour for vehicle that includes loading and unloading instructions at each station so that the resulting system is balanced.

In particular, it is difficult to balance the bike sharing system because it needs to solve the problem of the hybrid vehicle routing with simple goods in distribution (bicycles) based on the related value at the station [wikipedia, 2015].

\section{The Particle Swam Optimization Algorithm (PSO)}

A particle swarm is a population of particles, where each particle is a moving object that is through the search space and is attracted to previously visited locations with high fitness [kamali, 2014]. James Kennedy, a social psychologist, and Russell C. Abarhot, electrical engineer, are main provider of PSO algorithms. First, they had planned to develop a kind of computational intelligence by taking advantage of social models and social relations, which does not need a special individual abilities. Their first simulation was carried out in 1995 [Dorigo, 1996] [Bullnheimer, 1999], which led them to simulate the behavior of birds to find seed. This work was influenced Hepnter and Grnander, which 
was done as a nonlinear system to simulate the behavior of birds in 1990. Keney and Abarhot' Work led to the create a strong algorithm called particle swarm optimization or PSO for optimization [Olariu, 2006] [Siarry, 2006] [clerc, 2002].

PSO algorithm can be described as below.

- Step 1: Development of first location for every agent. Primary search point $\left(s_{i}^{0}\right)$ and speeds $\left(v_{i}^{0}\right)$ of every agent are created usually in valid area.

- Step2: evaluation of search point. value of objective function is calculated for each factor

- Step 3. Modifying any search point

- Step 4. Check output situation. If the current iteration number reaches a maximum number of predetermined iterations, then stop now, otherwise, go to step 2.

\section{Limitation programming algorithm $(\mathrm{CP})$}

Cp algorithm began by computer programmers in 1980. Its application fields is planning, sequencing, resource allocation, staff, and more.

Power of CP modeling:

* It does not require a linear constraints.

* It does not require special structures.

* Because it does not need to have a linear structure. It is displayed more directly.

* modeling is very easy for everyone (or knowledge is not necessary)

Optimization with CP:

Optimization process is as follows:

1- Finding a practical solution for $\mathrm{X}$

$2-\mathrm{Z}=\mathrm{C}(\mathrm{X})$

3- Adding limitation $\mathrm{C}(\mathrm{X})<\mathrm{Z}$

4- If a solution was found for $\mathrm{X}$

a) Go to step2

Otherwise

B) Return the value of the last $\mathrm{Z}$

Application:

Some $\mathrm{Cp}$ applications can be mentioned include:

* Combining CP with other methods

* Using issuing step on each node to solve MIP in B \& B algorithm 
Using the CP to finding neighbors in local search algorithms

* Using CP to convert the children in the intersection operators in genetic algorithm to cut-off points

* Finding an early solution in other ways

\section{Combination of limitation programming algorithms and swarm particle}

In the current paper we have presented a hybrid approach for balancing the bike sharing system based on limitation programming algorithm and particle swarm algorithms. Here we compare the results obtained in this paper and describes how to implement.

A) Model Definition

Introduced model is based on the limits i.e we have to satisfy a number of limitations. Limitations are: the number of bicycles, the number of citizens and shifting system that define the system as follows:

Algorithm 1. PSO + CP

Input: $\mathrm{X}=\mathrm{XAnts} \cup \mathrm{XCP}$, a set of integer variables partitioned into variables

Dealt with $\mathrm{PSO}$ and $\mathrm{CP}$, respectively

$\mathrm{C}$, a set of constraints

$\mathrm{F}$, a cost function

Create Initial population

1 initialize all Particle trails to gbest;

$2 \mathrm{~g} \leftarrow 0$;

3 repeat

4 for $\mathrm{k} \in\{1, \ldots, \mathrm{n}\}$ do

$5 \mathrm{Ak} \leftarrow \varnothing ;$

6 repeat

7 select a variable $x i \in X$ population, so that $x i \_\in \operatorname{var}(\mathrm{Ak})$, and a value

$\mathrm{j} \in \mathrm{Dj}$ according to the Particle position $\tau \mathrm{ij}$ (and possibly the

heuristic information $\eta \mathrm{ij}$ );

8 add $\{\mathrm{xi}:=\mathrm{j}\}$ to $\mathrm{Ak}$;

9 if Propagate $(\mathrm{Ak}, \mathrm{C})=$ Failure then

10 Backtrack(Ak);

11 until $\operatorname{var}(\mathrm{Ak})=$ XAnts;

12 PSOsearch(X, C,F);

13 update Particle position using $\{\mathrm{A} 1, \ldots, \mathrm{An}\}$ and $\mathrm{F}$;

$14 \mathrm{~g} \leftarrow \mathrm{g}+1$;

15 until best-position(g,Ai, time);

b) limitation

We will start with routing limitations for paths.

C) Implementing the particle swarm algorithm

Then we created a particle of mass algorithm is as follows:

iterations $=40:$ the number of frequency to obtain Optimization of particle

inertia $=1.0$; density coefficient

correction_factor $=2.0$;

PSO_size $=49$; The initial population size 
$\mathrm{u}=0.2$; Particle learning rate

d) parameter development

We created the following parameters for defined issue

1- parmeters of citizens

Function Parameters include:

$\mathrm{T}$ : the time in seconds

D: distance variable

M: the number of bicycle number of stations $n$

At (action time) the valid time to work i.e. we have time to the value of deadline variable to meet created limitation.

El: variable to remove a citizen from allocating bike. (If a citizen is removed from the bicycle allocation, again it is at the queue of this shifting system . this process is so fast that he did not wait to get bikes).

Cost is cost function pso.

F: cost to meet limitation

E: the value of calculated error based on factors of occurred probabilistic error

The first function related to citizens (cost) receives the number of bicycles and stations and the distance between each station and at the el and calculates the cost function pso. The cost function can be selected from particles of a population that have more appropriate optimality. The second function called lockstations returns a value $t$ to get the parameters described above.

$\mathrm{t}$ is time of locking or lack of service or Lack of service stations. It causes bike over kept at the station and prevent the allocation of excessive bicycles. As you see, this action is repeated as a number of $\mathrm{m}$ and $n$, and each time it updates the value of $t$.

\section{The parameters of the bike stations}

According to the above description, the first function pso specifies costs to satisfy the limitaion. And the second function i.e. the bike stations, determines parameters of the input values if there are limitations to the service station for a time limit is to be resolved. The $t$ value is calculated from the following equation

$t(a t(i, j), a t(i, j+1))=(1-e) * t(a t(i, j), a t(i, j+1))+d t ; \%$ updating

h) problem

However, due to a development of problem and parameter coding will solve the problem using the following parameters. As you can see below, it solved satisfy limitation and in each station every bike are allocated. 


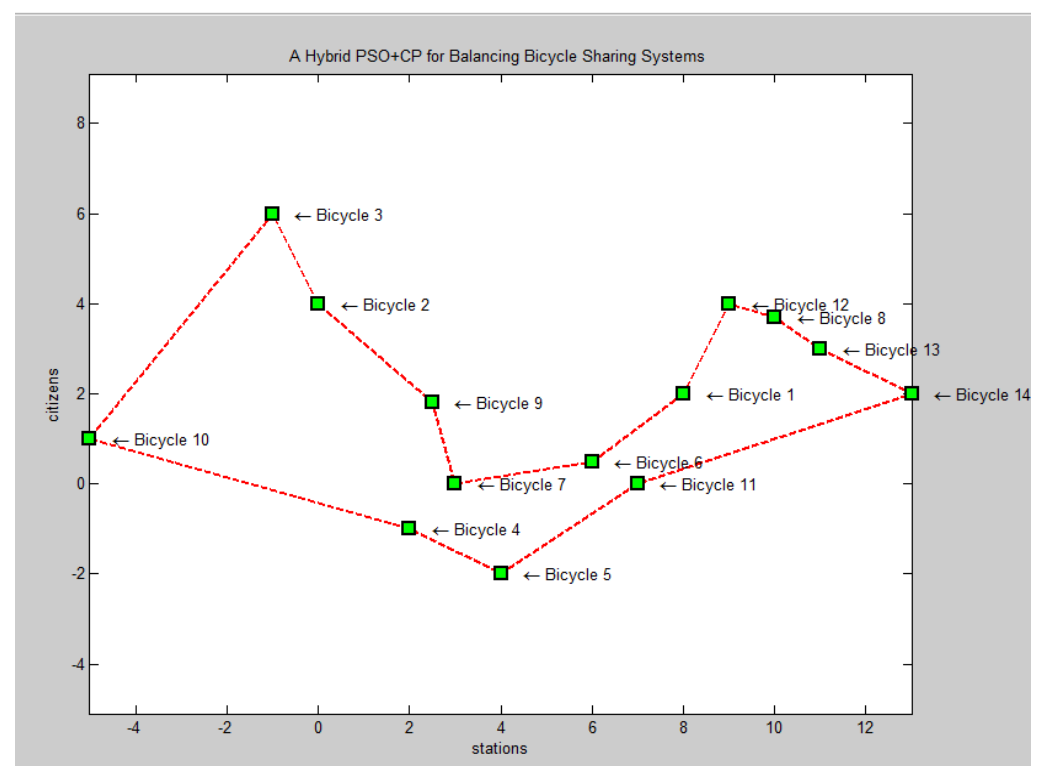

Figure 1. Show the bike Station

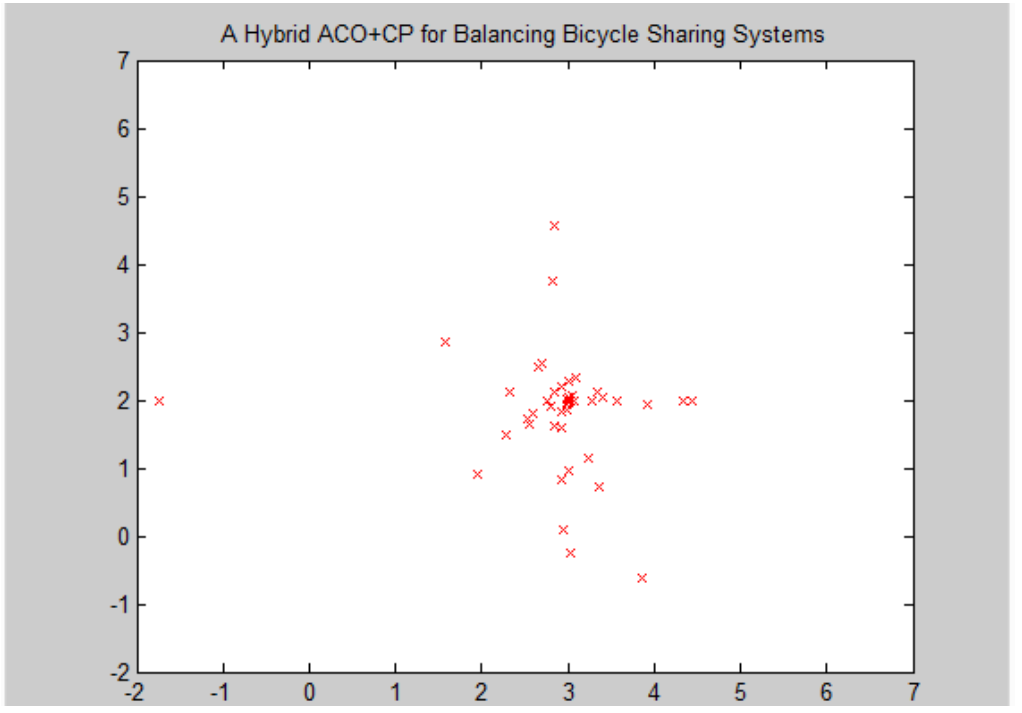

Figure 2. Solved Satisfy limitation

v) evaluation and compare

im this chapter, first we determine initial values that as follow :

1- all experiments were performed on a system with $3 \mathrm{~GB}$ of RAM and a $2.24 \mathrm{GHz}$ Intel CPU.

2- We chose the initial values pso

3- While graphical display particles move to the optimal point

We take size of the population as 49 that the size of the particles are considered to be equal $7 * 7$,so the size of swarm population is 49 . 


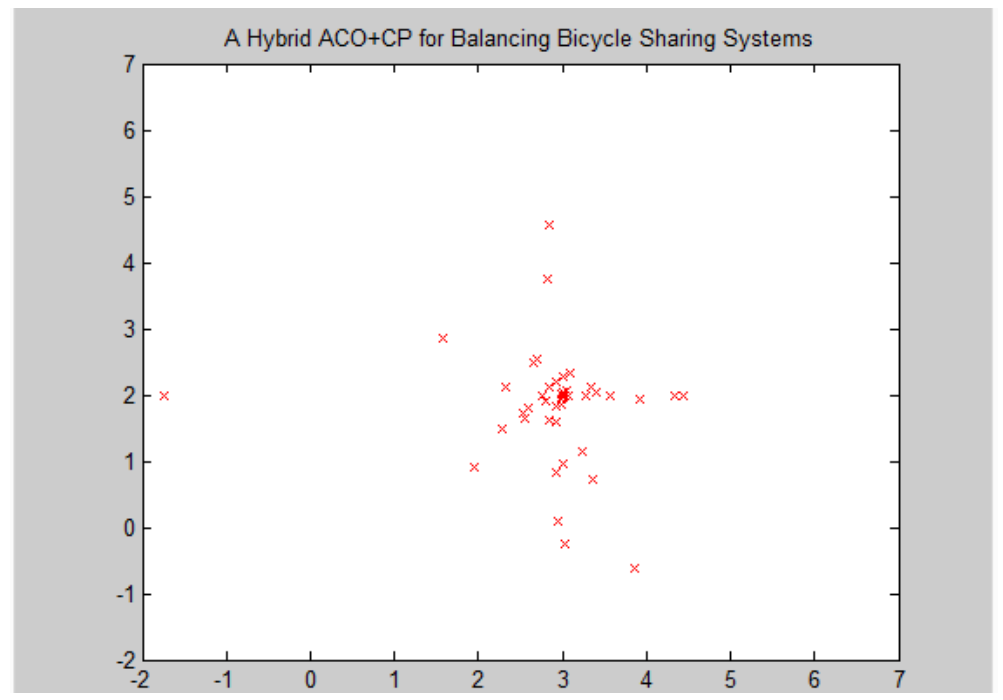

Figure 3. shows that problem can be solved easily by increasing number of station and response time reduce under 50 seconds.

According to solving above problem, proposed algorithm is computed as follow.

Our diagram shows that problem can be solved easily by increasing number of station and

Response time reduce under 50 seconds. Our graph that obtained in repeated time, time continues to 120 seconds. This shows the superiority of the proposed method. I refer you to the final output that final $\mathrm{t}$ is calculated about 19 seconds.

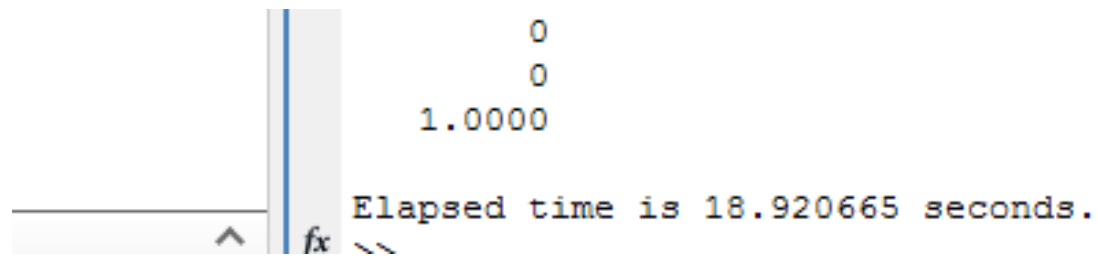

Also, in worst condition, average runtime lead to $80 \mathrm{sec}$ that is low for number of stations and is better than aco method.

Paper digraph also solve problem but runtime will continue to $120 \mathrm{sec}$.

As you observe above, whatever number stations increases, so the the algorithm efficiency will increases. According to the chart, the pso is the better method than our method.

\section{Acknowledgement}

The author is highly thankful to the referee for his valuable suggestions for improving the quality of the paper. 


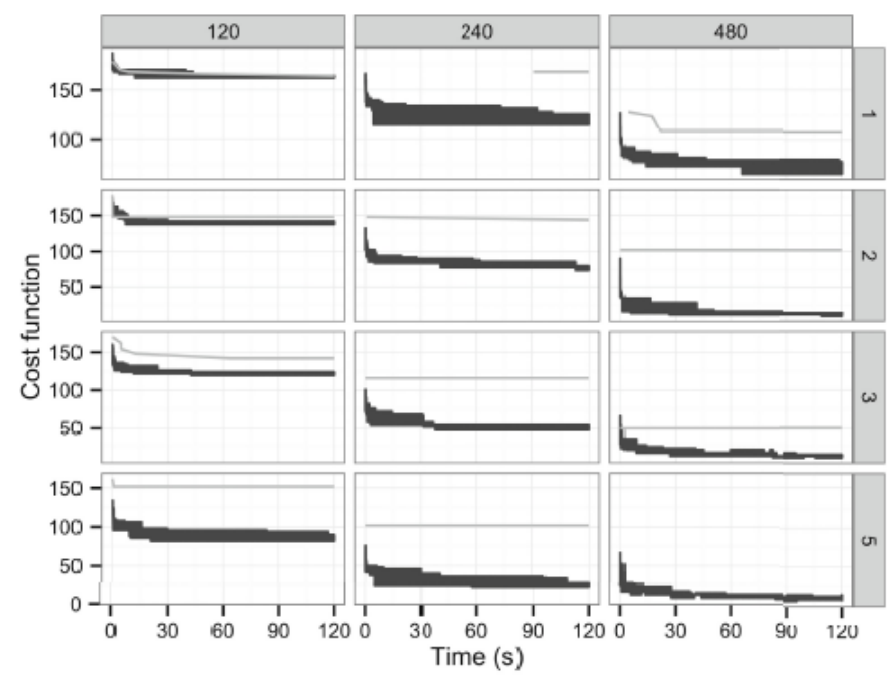

Figure 4. Shows a comparison between the $\mathrm{ACO}+\mathrm{CP}$ (stretch and warp) and CP (the thin and light) on the distance issue, with 30 stations, matrix charts Column, the time validity of the vehicle and number of available vehicle rows [Blesa, 2013].

\section{References}

[1] B. Bullnheimer, R. Hartel, C. Strauss, "A new rank-based version of the ant system: A computational study, Central European Journal of Operations Research and Economics", no. 7 (1999), pp. 25-38.

[2] F. Fadaie, J. Vahidi, "The optimal Bicycle Time sharing systems By Hybrid genetic algorithm and ant colony", The second National Conference on applied research in computer science and information technology, (2015), 26 Feb.

[3] M. Dorigo, V. Maniezzo, A. Colorni, "The Ant System: Optimization by a colony of cooperating agents", IEEE Transactions on Systems, Man and Cybernetics, part B, no. 26 (1996), pp. 29-41.

[4] M. Clerc, J. Kennedy, "The Particle Swarm: Explosion, Stability and Convergence in multidimensional complex space", IEEE Transactions on Evolutionary Computation, vol 20, no. 6 (2002), pp. 58-73.

[5] María J. Blesa, Christian Blum, Paola Festa, Andrea Roli, Michael Sampels (Eds.) "Hybrid Metaheuristics", $8^{\text {th }}$ International Workshop, HM 2013,Ischia, Italy, May (2013),Proceedings.

[6] P. Siarry, "Metaheuristics for Hard Optimizations", Springer-Verlag Berlin Heidelberg, (2006).

[7] S. Olariu, A.Y. Zomaya, "Handbook of Bioinspired Algorithms and Applications", Taylor \& FrancisGroup, LLC Press, (2006).

[8] S.Kamali, "Portfolio Optimization using Particle Swarm Optimization and Genetic Algorithm", vol 10 (2014), pp. 85-90.

[9] Wikipedia. "Bicycle_sharing_system". http://en.wikipedia.org., January (2015). 